\title{
Capacitance metrology at the BIPM to support National Metrology Institutes
}

\author{
J. Angel Moreno $^{1^{*}}$ and Pierre Gournay ${ }^{1}$ \\ ${ }^{1}$ BIPM, Pavillon de Breteuil, 92312 Sèvres, France
}

\begin{abstract}
The Bureau International des Poids et Mesures (BIPM) is an international organization which works to promote and advance a coherent system of measurements throughout the world, traceable to the International System of Units (SI). One of its activities consists of the dissemination of units and the comparison of national measurement standards, which requires the availability of well-known and well-characterized standards. In particular, in the field of electricity, the BIPM supports capacitance measurements based on a quantum reference, impedance bridges and stable transfer standards, establishing traceability to the defining fundamental constants of the SI. In this paper it will be explained how the farad is realized at the BIPM, including a general description of the measuring systems, and how this unit is maintained.
\end{abstract}

\section{Introduction}

The Bureau International des Poids et Mesures (BIPM), located at Sèvres, France, is an international organization focused on promoting and advancing the global comparability of measurements, by working together with the National Metrology Institutes (NMIs) of its member states and the Regional Metrology Organizations (RMOs).

To reach its objectives, the BIPM coordinates comparisons of national measurement standards, which ensure the international acceptance of measurement results, producing a high impact on important areas of science, innovation, international trade and quality of life, among others [1]. Additionally, the BIPM maintains properly selected key reference standards, particularly in the field of mass, chemistry, ionizing radiation and electricity, to provide calibration services for many other reference standards maintained by the NMIs in the entire world.

The Physical Metrology Department of the BIPM has a work program devoted to the realization and maintenance of three important electrical units: the volt, the ohm and the farad [2]. The volt and the ohm are realized directly with high accuracy by means of the wellknown Josephson and Hall quantum effects, respectively, but in the case of the farad its realization can be made in two different ways as described below.

\footnotetext{
* Corresponding author: angel.moreno@bipm.org
} 


\section{Realization of the farad}

The farad is the unit of the electrical capacitance. This quantity is extensively used in the development of electric and electronic devices for a large diversity of industrial and scientific applications. It is also used to measure indirectly other quantities through capacitive transducers as, for instance, capacitive displacement sensors, MEMS capacitive accelerometers, or even high voltage capacitive dividers. Because of the importance of the farad, it is maintained at many NMIs at a high level of accuracy to provide strong traceability to support all these applications.

The realization of the farad with high accuracy is not easy to achieve. In the framework of the International System of Units (SI), there are two recognized primary methods to realize the farad consisting of [3]:

a) the use of a calculable capacitor, which is an arrangement of finely aligned electrodes placed in vacuum, where the capacitance is determined by means of the value of the vacuum electric permittivity $\varepsilon_{0}$ and measurements of length $\Delta_{\ell}$ according to the equation $1[4]$, and

b) the comparison of the impedance of a capacitor with the impedance of a resistor at a fixed frequency, using an electrical circuit known as Quadrature Bridge [5], where the capacitance can be determined by means of the value of the resistor, known in terms of the von Klitzing constant $R_{\mathrm{K}}$, which is directly related with the Planck constant $h$ and the elementary charge $e$.

$$
C=\frac{\varepsilon_{0} \ln 2}{\pi} \Delta_{\ell}
$$

Both primary realizations have been developed by the BIPM not only to support capacitance services for the NMIs of the member states, but additionally, they will be used to confirm consistency between the quantum reference (quantum Hall effect) and the classical one (calculable capacitor) [6]. Up to now, the realization of the farad for services at the BIPM has been based on the quantum Hall effect, which will be described hereinafter.

\section{Traceability chain of the farad at BIPM}

As can be seen in the figure 1, at the BIPM the origin of the traceability of the farad is the Quantized Hall Resistance (QHR), whose value is defined by means of the von Klitzing constant $R_{\mathrm{K}}=h / e^{2}$ where the numerical values of the Planck constant $h=6.62607015 \times 10^{-34}$ $\mathrm{J} \mathrm{s}$, and the elementary charge $e=1.602176634 \times 10^{-19} \mathrm{C}$ are fixed in the SI [7]. Using the second plateau of the QHR quantization curve, the resistance value of the QHR is $R_{H}=R_{K} / 2$ $=12.90640373 \mathrm{k} \Omega$, rounded for a relative resolution of $1 \times 10^{-9}[8]$. 


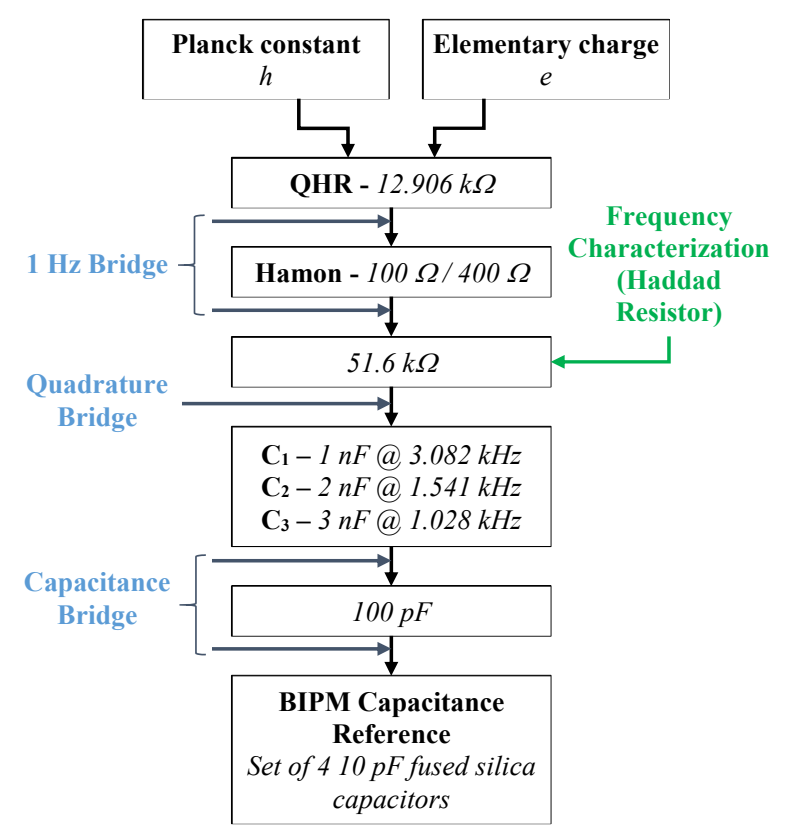

Fig. 1. Traceability chain of the farad at BIPM.

By means of a low frequency resistance comparison bridge operated at $1 \mathrm{~Hz}$, based on a room temperature current comparator with nominal ratio of $1: 129$, two $51.6 \mathrm{k} \Omega$ resistors are calibrated against the QHR using as transfer standard a Hamon resistance network. Such a network has the property of maintaining its relative error from its nominal value either in parallel configuration for $100 \Omega$ or in series configuration at $400 \Omega$.

At this point, the quadrature bridge allows the direct comparison of the calibrated $51.6 \mathrm{k} \Omega$ resistors and different arrangements of $1 \mathrm{nF}$ capacitors at fixed frequencies, according to equation 2.

$$
2 \pi f R C=1
$$

Because the value of the $51.6 \mathrm{k} \Omega$ resistors is known at $1 \mathrm{~Hz}$, it is strictly necessary to determine their frequency correction from $1 \mathrm{~Hz}$ up to the usual max operating frequency of $3 \mathrm{kHz}$. This is determined using as reference a Haddad coaxial resistor, which has negligible frequency dependence below $10 \mathrm{kHz}$, and a four-terminal-pair (4TP) coaxial bridge. Finally, using a capacitance coaxial bridge with ratio $1: 10$, the four $10 \mathrm{pF}$ capacitors of the BIPM capacitance reference are calibrated using $100 \mathrm{pF}$ capacitors as transfer standards.

It is very important to remark that the main inductive voltage dividers (IVD) used in the ratio bridges are periodically calibrated using a bootstrap method [5]. 


\subsection{Frequency characterization of resistors}

A key task in the traceability chain of the farad at the BIPM is the characterization of the frequency dependence of the $51.6 \mathrm{k} \Omega$ resistors. This is realized following the scheme shown in the figure 2. In a first step, using as reference a Haddad coaxial resistor, the changes with frequency of the value of a $12.906 \mathrm{k} \Omega$ transfer resistance standard is characterized by means of a 4TP bridge configured in a 1:10 measurement ratio. In a second step, this transfer standard is used to characterize the $51.6 \mathrm{k} \Omega$ resistors using the same 4TP bridge configured in a 1:4 measurement ratio.

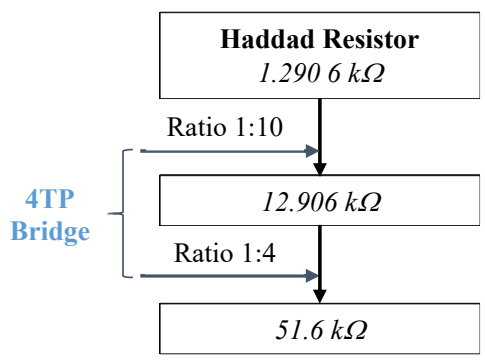

Fig. 2. Measurement scheme for frequency characterization of the $51.6 \mathrm{k} \Omega$ resistors.

The characterization of the resistors is based on differential measurements $\Delta_{R f}$ obtained from the resistance value measured at a pivot frequency $f_{0}$ and the resistance value measured at different frequencies, as it is shown in the figure 3. Using these measurements, it is possible to find the resistance difference $\Delta_{R I H z}$ by statistical extrapolation, which represents the change of the resistor from $f_{0}$ to $1 \mathrm{~Hz}$. Additionally, $\Delta_{R I H z}$ can be obtained through direct measurements by using the $1 \mathrm{~Hz}$ bridge and the 4TP bridge, operating them in an alternated way.

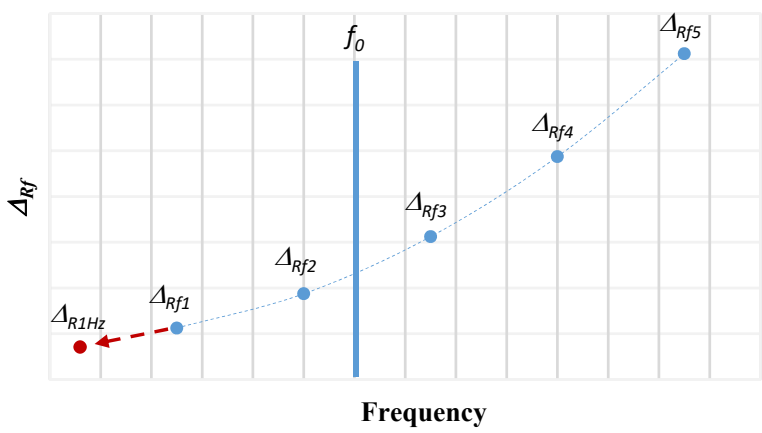

Fig. 3. Measurement scheme for frequency characterization of resistors.

Because all the $\Delta_{R f}$ measurements are referred to $f_{0}$, it is possible to compute the change of the resistor from $1 \mathrm{~Hz}$ to any other frequency, where the values of particular interest are 1028 $\mathrm{Hz}, 1541 \mathrm{~Hz}$ and $3082 \mathrm{~Hz}$, as illustrated in figure 4. 


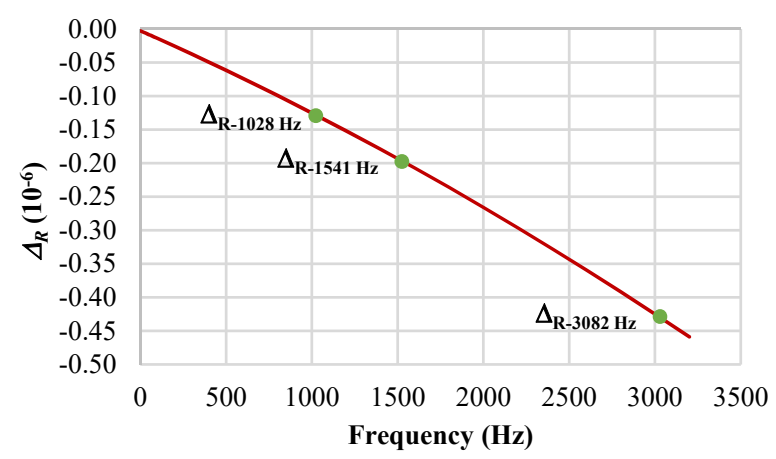

Fig. 4. Typical characterization curve of the $12.906 \mathrm{k} \Omega$ and $51.6 \mathrm{k} \Omega$ resistors used at BIPM.

\subsection{Brief description of impedance bridges}

Because the impedance bridges play a very important role in the implementation of the chain of traceability, we explain in the following in a simplified way their functioning principle.

\subsection{1 $1 \mathrm{~Hz}$ bridge}

As can be seen in figure 5, the circuit of the $1 \mathrm{~Hz}$ bridge is based on a current comparator with a measurement ratio defined by the number of turns of the windings $N_{l}$ and $N_{2}$, which in this case is $1: 129.0625$. Since the ratio windings of the comparator are formed by strands of guarded cables connected in series, a very low relative winding turns ratio error can be obtained [9], of the order of 1 part in $10^{9}$.

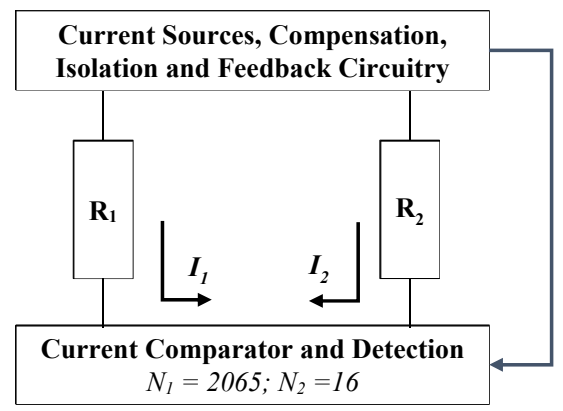

Fig. 5. Basic scheme of the $1 \mathrm{~Hz}$ bridge.

At balance, the $1 \mathrm{~Hz}$ currents $I_{1}$ and $I_{2}$ produce opposite magnetic fields in the comparator, in such way that a zero field is present in the detection coil. The result of the measurement is the difference from the nominal ratio $\delta_{R}$ expressed as in the equation 3 . In such way, it is possible to obtain the value of one resistor knowing the value of the other, with relative uncertainty of about 1 part in $10^{9}$.

$$
R_{1} / R_{2}=2065 / 16\left(1+\delta_{R}\right)
$$




\subsubsection{Quadrature bridge}

Because of its relative complexity, the figure 6 shows only the electrical concept of this bridge for the particular case of the comparison of a single resistor with a single capacitor. A complete description of this bridge can be found in [5].

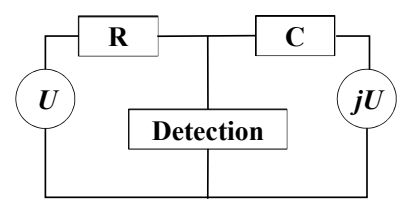

Fig. 6. Electrical concept of the quadrature bridge.

In this circuit, the voltages $U$ and $j U$ have the same frequency $f$ but differ in phase by $90^{\circ}$. When the frequency is varied, the impedance of the capacitor $Z_{C}=1 /(2 \pi f C)$ can be made equal to the value of the resistor $R$ inducing a zero voltage in the detection arm. Under these conditions, the value of the capacitor can be found simply with the equation 4 .

$$
C=1 /(2 \pi f R)
$$

In addition to this simple principle circuit, it is necessary to use several other circuits mainly based on IVDs to produce the voltage $j U$ and to balance the bridge. The calibration of the $1: 1$ main IVD (not shown) is not required, but it is necessary to make a second measurement inverting the connections of the bridge in order to properly eliminate the ratio error of this divider, obtaining the value of the capacitors with a relative uncertainty of $10^{-9}[10]$.

\subsubsection{Capacitance bridge}

This two-terminal-pair (2TP) coaxial comparison bridge is based on a calibrated standard IVD with ratio 1:10, as shown in figure 7 . In a similar way as in other designs [11], in-phase and quadrature currents are injected in the $\mathrm{C}_{1}$ or $\mathrm{C}_{2}$ arm using additional IVD circuits in order to obtain zero voltage in the detection arm. Under these conditions, the ratio of the impedance of the two capacitors is equal to the standard voltage ratio of the standard IVD.

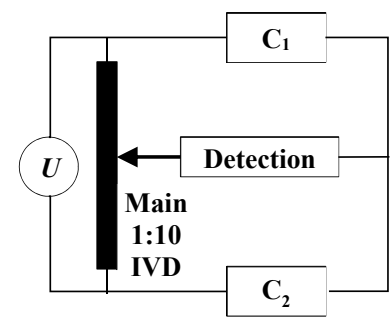

Fig. 7. Basic scheme of the capacitance bridge. 
At balance, the measurement results can be expressed as the equation 5, where $\delta_{C}$ is the difference from the nominal ratio, so that it is possible to obtain the value of one capacitor knowing the value of the other, with relative uncertainty of parts in $10^{9}$.

$$
C_{2} / \mathrm{C}_{l}=\left(1+\delta_{C}\right)
$$

\subsubsection{TP resistance comparison bridge}

This last bridge used in the traceability chain follows the same concept as the 2TP capacitance bridge. However due to the 4TP configuration of the resistance standards some additional balancing sub-circuits are required.

Figure 8 shows the basic scheme of the bridge, where it can be noted that the terminals of the resistors require to be unequivocally defined and that additional compensation circuits need to be included to null the current in the bridge arms linking $R_{1}$ and $R_{2}$ to the main standard IVD.

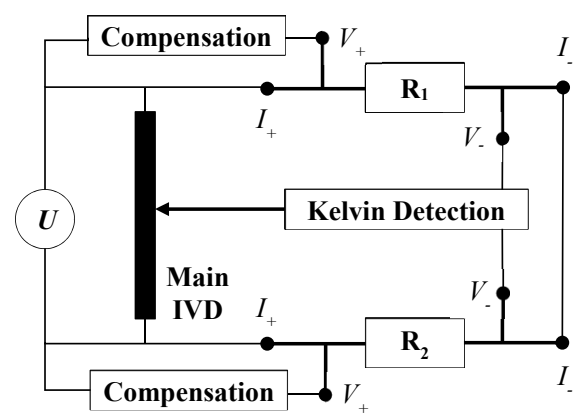

Fig. 8. Basic scheme of the 4TP bridge.

The detection circuit uses a combining network which follows the concept of a "Kelvin double bridge" [5] to compensate for the impedance of the connecting cable between the $I$ terminals of the resistors, which could induce a non-negligible voltage drop in that cable. At balance, the measurement results can be expressed as in equations 6 and 7 for the cases of the ratio measurements 1:10 and 1:4 respectively, where $\delta_{R}$ represents the difference of the relative error from the nominal value of the corresponding resistors under measurement $\varepsilon_{R l}$ and $\varepsilon_{R 2}$, respectively, as expressed in the equation 8 . This last equation can be obtained with a relative uncertainty of $10^{-9}$.

$$
\begin{gathered}
\mathrm{R}_{1} / \mathrm{R}_{2}=10\left(1+\delta_{R}\right) \\
\mathrm{R}_{1} / \mathrm{R}_{2}=4\left(1+\delta_{R}\right) \\
\delta_{R}=\varepsilon_{\mathrm{R} 1}-\varepsilon_{\mathrm{R} 2}
\end{gathered}
$$




\section{Maintenance of the farad}

As many passive standards, the four $10 \mathrm{pF}$ fused silica capacitors of the BIPM capacitance reference change due to many factors inducing typical slow and predictable drifts. Due to the rather high temperature coefficient of fused silica, the capacitors are placed in an oil bath at a nominal temperature of $25^{\circ} \mathrm{C}$ with a temperature stability of the order of the $\mathrm{mK}$. This bath is located in a closed area with controlled temperature of $23{ }^{\circ} \mathrm{C}$. The temperature of the capacitors is measured using individual platinum sensors and a thermometry bridge, which assure small corrections with relative uncertainty in the order of $10^{-9}$.

The group of capacitors is usually calibrated against the QHR two times per year by means of the scheme described in the figure 1, but it is possible to calibrate them at any moment using one of the reference resistors maintained at the BIPM resistance laboratory. The BIPM capacitance reference value is defined as the average capacitance of the group, which is more stable (lower drift) than the capacitance of the four individual capacitor [12]. In order to maintain a permanent surveillance between each calibration, the capacitors are compared periodically in a star scheme using the 1:10 ratio capacitance bridge as shown in figure 9, where a $100 \mathrm{pF}$ capacitor is used as common reference.

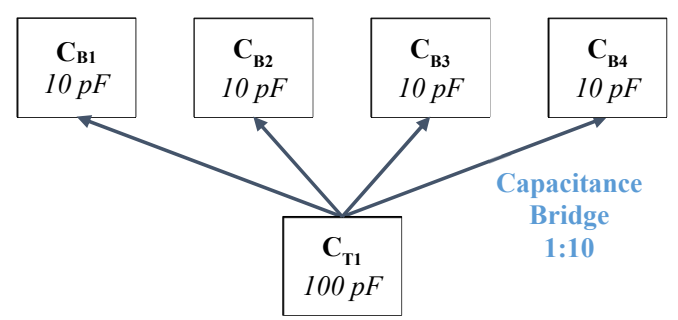

Fig. 9. Star comparison of capacitors.

This control scheme allows the detection of possible changes or deviations from the expected trend for any of the capacitors with respect to the average value or with respect to the other capacitors, including the $100 \mathrm{pF}$ capacitor, which is also maintained under surveillance and acts as a backup or reference standard if necessary.

Based on the techniques explained above, the BIPM is able to provide capacitance calibration services to NMIs with standard relative uncertainties in the order of $10^{-8}$, considering all the contributions in the traceability chain and the repeatability of the capacitor under calibration.

In addition to the capacitance value, given at a frequency of $1592 \mathrm{~Hz}$, a study on frequency and voltage dependence is provided for each calibrated capacitor. This study is realized using the known frequency dependence of the capacitance reference from $1 \mathrm{kHz}$ to $3 \mathrm{kHz}$, and the prior characterization of two capacitors from different manufacturers with nominal values of $10 \mathrm{pF}$ and $100 \mathrm{pF}$, whose ratio has negligible voltage change from $50 \mathrm{~V}$ to $100 \mathrm{~V}$. This provides the NMIs with all the information to support and maintain their national references properly and disseminate the farad to secondary laboratories and finally to the industry. 


\section{Conclusions}

At the BIPM sophisticated measurement systems are used to provide proper traceability of the farad to the defining fundamental constants of the SI, mainly through the quantum Hall effect. These measuring systems consist of a set of impedance bridges which allow suitable comparison between well characterized standards, whose stability is kept under permanent surveillance through periodic calibrations and verifications.

For many NMIs in the world which do not possess their own primary standards of capacitance, the BIPM calibration services are the starting point for disseminating the farad to secondary levels and finally to the industry, where this work can finally produce impact and benefits. Additionally, the BIPM organizes international measurement comparisons with NMIs which realize the farad, which help them to validate and demonstrate their measurement capabilities.

\section{References}

1. BIPM, https://www.bipm.org/en/cipm-mra/, June 2019.

2. BIPM, https://www.bipm.org/en/bipm/physmet/, June 2019.

3. CCEM, https://www.bipm.org/utils/en/pdf/si-mep/SI-App2-ampere.pdf, May 2019.

4. K. Jones and A. C. Corney, Metrologia 24, 1-11 1987.

5. B.P. Kibble and G.H. Rayner, Adam Hilger Ltd, Bristol, 1984.

6. P Gournay, N. Fletcher, L. Robertson and M. Stock, CPEM Conf. Dig. 2016.

7. BIPM, https://www.bipm.org/en/measurement-units/, June 2019.

8. CODATA, https://physics.nist.gov/cgi-bin/cuu/Value?rk, June 2019.

9. F. Delahaye and D. Bournaud, IEEE Trans. Instrum. and Meas., Vol. 42, n², 1993.

10. F. Delahaye and R. Goebel, IEEE Trans. Instrum. and Meas., Vol. 54, n², 533, 2005.

11. J.A. Moreno and R. Hanke, CPEM Conf. Dig. 2000.

12. W. G. Eicke and J. M. Cameron, Tech. note 430, NBS 1967. 